\title{
Participación de la población rural en los procesos territoriales de desarrollo
}

\section{Rural Participation in the Territorial Development Processes}

\author{
Patricia María Bartol de Imbach ${ }^{7}$ \\ Geolatina S.A. \\ Turrialba, Costa Rica \\ patoimbach@gmail.com
}

Recibido 21 de mayo de 2012 • Corregido 02 de octubre de 2012 • Aceptado 07 de noviembre de 2012

Resumen. Este documento es un espacio de reflexión acerca de la necesidad y la importancia de asumir el compromiso de crear un puente entre la participación social y la construcción de capacidades ciudadanas. El diálogo permanente del saber experto y el saber local debe alimentar los procesos de aprendizajes pertinentes y relevantes desde el contexto rural de la región centroamericana y hacia esta. La coherencia y persistencia de estos procesos permitirá promover el bienestar humano, a partir de los cambios experimentados en la ruralidad. En el territorio centroamericano existen numerosas iniciativas que, para concretar sus propuestas, requieren de la participación social e institucional efectiva. La educación, en sus diversas manifestaciones y con sus variados recursos, tiene una gran responsabilidad en posibilitar el aprovechamiento de estas por parte de la ciudadanía.

Palabras claves. Educación rural, participación, calidad de la vida, desarrollo rural, diálogo de saberes.

Abstract. This paper discusses the urgency of creating a bridge between social participation and civic capacity building. The permanent dialogue between expert and local knowledge should sustain significant, relevant learning processes from/to the rural areas of the Central American region. Consistency and persistence of these processes will enhance human welfare based on the changes experienced in the rural areas. Numerous Central American initiatives require effective social and institutional participation to be implemented. Education, in its different forms and through its different resources, has the crucial responsibility of helping citizens to take advantage of those initiatives.

Keywords. Rural education, participation, quality of life, rural development, knowledge dialogue.

\footnotetext{
Argentina, residente en Costa Rica, Máster en Educación Rural Centroamericana de la Universidad Nacional, Costa Rica.
} 
Vivir es aprender, aprender es cambiar, y cambiar es vivir aprendiendo en interacción con el contexto (...)

(De Souza, 2005, p. 12)

La realidad de las comunidades rurales de Centroamérica experimenta, desde finales del siglo XX, cambios que la afectan de diversa forma. Estos cambios, asociados a diversas actividades y recursos comunitarios, modifican las manifestaciones culturales y sociales $y$, a la vez, generan repercusiones en la identidad de las poblaciones rurales. En algunas circunstancias, el bienestar humano y el de los ecosistemas se ven beneficiados y; en otros, por el contrario, son deteriorados. Los Estados de la región han reducido sus presupuestos, con el creciente debilitamiento y privatización de los servicios públicos, y con la implementación, con frecuencia, de iniciativas de descentralización administrativa. Otro elemento importante lo constituyen las distintas políticas y estrategias puestas en marcha en Centro América y el Caribe, las que atienden con especial interés el carácter rural de la región.

Estos cambios tienen origen en decisiones que se toman más allá de los territorios rurales, aunque otros dependen directamente de los habitantes de estos.

En conclusión, se están produciendo cambios sociales, económicos, políticos y ecológicos que afectan a la agricultura y al medio rural y que definen también, nuevas demandas de la sociedad y el surgimiento de una nueva estructura de oportunidades, la cual es percibida de manera distinta por cada uno de los países, en especial, atendiendo a las diferencias que marcan los distintos niveles de desarrollo. (Instituto Interamericano de Cooperación para la Agricultura [IICA] y Dirección de Desarrollo Rural Sostenible, 2000, p. 8)

Esta relación dialéctica entre los territorios rurales con los contextos más allá de sus fronteras ha configurado lo que se denomina la "Nueva ruralidad".

Crece, con ello, la necesidad de la participación de la ciudadanía -informada y reflexivaque permita asumir la responsabilidad de influir en los procesos de transformación en marcha. La educación a través de todas sus manifestaciones y recursos ocupa un lugar ineludible en la construcción de capacidades que permitan la toma de decisiones en forma efectiva, incluyente y equitativa por parte de todos los actores rurales.

El protagonismo en la construcción del presente y futuro de los territorios rurales reside en sus propios habitantes. Dadas las oportunidades que se abren, se requieren procesos que 
permitan construir una nueva ciudadanía, informada, comprometida y activa. Desde múltiples iniciativas se debe aportar a la construcción de un diálogo entre los saberes locales y los que aportan quienes desempeñan funciones técnicas y políticas más allá del contexto local. El bienestar de la calidad de la vida humana y de los ecosistemas que la hacen viable solo se construirá si este diálogo se mantiene y revisa, lo cual permitiría la construcción de aprendizajes a partir de la práctica.

\section{Descripción de la situación}

\section{¿Qué está pasando en los territorios rurales?}

Los habitantes rurales de la región centroamericana han experimentado transformaciones en sus territorios que no siempre han significado una mejor calidad de vida ni humana ni de los ecosistemas.

A partir de la década de los 80, la reestructuración del Estado ha significado el recorte y la disminución de la eficiencia en la prestación de algunos servicios. Se ha llegado, en algunas ocasiones, a la privatización de la prestación de servicios estratégicos tales como la salud y la educación, los cuales se transforman en mercancías que se rigen por la lógica del mercado y no por el bienestar humano. Esta situación abre brechas discriminatorias en el bienestar para quienes no están en condiciones de pagar por el servicio. Es así como las autoridades democráticas pierden credibilidad (Kliksberg, 2011) y dejan de responder al mandato de quienes representan.

Se han abierto procesos de descentralización de la administración del Estado, con lo que se ofrece una oportunidad para la participación ciudadana. Sin embargo, es poco frecuente que estas oportunidades sean acompañadas de procesos de construcción de las competencias necesarias para una implementación exitosa.

El debilitamiento de las democracias influye negativamente en el tejido social. Las relaciones sociales se transformaron y alteraron, paulatinamente, los valores de confianza y credibilidad. Nuevos actores sociales surgen como respuestas a incentivos o proyectos, pero con facilidad pierden el rumbo o se desvanecen. En algunas ocasiones las migraciones permanentes o transitorias aportan costumbres y valores nuevos, los cuales, si no son críticamente considerados, pueden atentar contra la cohesión social.

La producción en los territorios rurales vive la influencia de las dinámicas de las economías nacionales e internacionales. Estos cambios, sumados a otras variables, exigen reconfiguraciones de la actividad productiva. Se introducen nuevas tecnologías productivas, en algunos casos solo al alcance de quienes cuentan con capital suficiente para implementarlas. El intercambio con mercados internacionales determina la necesidad de diversificar las actividades económicas rurales, incorporando algunos rubros como turismo, procesamiento post cosecha, producción para la exportación y otros. Debido a esta nueva configuración de la producción agropecuaria 
-más al servicio de la macroeconomía que de las necesidades humanas locales-, la seguridad ${ }^{2}$ y la soberanía alimentaria ${ }^{3}$ se constituyen en aspectos polémicos.

La calidad y cantidad de los recursos naturales y los servicios que se prestan han sido revalorizadas, debido a la escasez en las habituales fuentes de suministro. Las condiciones de algunos recursos considerados "inagotables" hasta hace poco tiempo nos hacen mirar al futuro con preocupación. Los cambios son acelerados, las grandes potencias industrializadas vuelven la mirada hacia los territorios rurales, posibles proveedores de bienes necesarios para sus industrias. El interés súbito en las explotaciones mineras y en la compra de tierras agrícolas en toda América Latina, por parte de empresas transnacionales, en la última década, son una evidencia de lo planteado, así como la secuela de numerosos conflictos sociales con las poblaciones rurales afectadas (Grain, 2010; Grain, 2012).

En algunos casos, las comunidades rurales se dividen ante las ofertas sustanciosas por la compra o explotación temporal de sus bienes. Los servicios que prestan los ecosistemas rurales cobran inusitado valor para todos los seres humanos, debido al deterioro de ciertas condiciones ambientales. Son frecuentes los desarrollos urbanísticos en zonas rurales. Estas urbanizaciones articulan las necesidades de campesinos impactados por la nueva economía y el deseo de los habitantes de las ciudades de vivir en ambientes más saludables.

La combinación de estas nuevas condiciones se refleja en cambios en la identidad de los territorios rurales. La institucionalidad, la organización social, las nuevas prácticas productivas, las migraciones, los recursos naturales; todo se combina y proporciona un nuevo significado a la ruralidad. Los cambios encuentran su expresión en transformaciones de las actitudes y valores que se concretan en prácticas, en algunos casos, insostenibles.

\section{¿Quiénes deciden estos cambios sobre la vida de las poblaciones rurales?}

Pese a la importancia de estos cambios para la calidad de la vida en los territorios, rara vez las decisiones son acompañadas por procesos participativos, equitativos e incluyentes de las poblaciones afectadas. Muchas de las decisiones son tomadas en centros de poder alejados de la realidad rural, la cual será la que vivirá las consecuencias. En otros casos son adheridas en forma poco reflexiva o informada por algunos representantes locales o territoriales.

\footnotetext{
La disponibilidad física de alimentos (...). El acceso de las personas a los alimentos (...). El logro de un nivel de bienestar nutricional (...). La estabilidad del acceso a alimentos adecuados en todo momento (...)" (ICA, s. f., párr. 2-5)

3 La soberanía alimentaria defiende el derecho de los pueblos a alimentos nutritivos y culturalmente adecuados, accesibles, producidos de forma sustentable y ecológica, y el derecho a decidir su propio sistema alimentario y productivo. Sitúa a aquellos que producen, distribuyen y consumen alimentos en el corazón de los sistemas y políticas alimentarias, por encima de las exigencias de los mercados y de las empresas. Defiende los intereses de e [sic] incluye a las futuras generaciones. Ofrece una estrategia para resistir y desmantelar el comercio libre y corporativo y el régimen alimentario actual, para encauzar los sistemas alimentarios, agrícolas, pastoriles y de pesca hacia su gestión por productores y productoras locales (...) (Soberanía Alimentaria, s. f., párr. 1)
} 
Suele ocurrir que las dinámicas se combinan o tienen lugar con una rapidez tal que tornan difícil que las instituciones reguladoras anticipen los resultados. Es así como se llega a dejar de lado posibles oportunidades o amenazas que se concretan al mediano o largo plazo. Esto suma el descrédito de la institucionalidad y debilita la vida democrática.

En el contexto de la región centroamericana, existen diversas instituciones, estrategias, políticas y programas. Estas iniciativas promueven oportunidades de articulación en la región a partir de los rasgos culturales y naturales compartidos, y a la vez diversos, que la caracterizan.

La región cuenta con el Sistema de Integración Centroamericana (SICA), integrado por los países de la región a los que se suma República Dominicana. A partir de la historia vivida, se reconoce la interdependencia del bienestar de los países que la integran. Los objetivos fundamentales del SICA abordan la multidimensionalidad del bienestar humano. Desde esta perspectiva, el SICA se compromete a promover la democracia, seguridad, libertad ciudadana, bienestar y justicia económica, economía, autodeterminación y desarrollo sostenible. Con estas responsabilidades asumidas se crearon órganos, instancias de seguimiento, secretarías e instituciones especializadas.

Estos enfoques fundamentan estrategias y políticas regionales. Algunas de ellas son la Estrategia regional ambiental y de salud (ERAS), Estrategia centroamericana de desarrollo rural territorial (ECADERT), Política agrícola centroamericana (PACA), Política de integración de pesca y acuacultura de Centro América, Programa estratégico del manejo de ecosistemas forestales (PERFOR), Programa regional de seguridad alimentaria y nutricional de Centro América (PRESANCA). El éxito de todas ellas depende de la participación social comprometida e informada. En la mayoría de los casos se deben desarticular paradigmas conceptuales de larga data y construir nuevas percepciones de la realidad comunitaria y regional. La difusión de información y la construcción de capacidades asociadas a los nuevos enfoques deben contemplar diversidad de medios, aunque todos ellos han reflexionar acerca del marco epistemológico del cual parten.

Las metas propuestas para mejorar y sostener las diversas dimensiones (Estrategia centroamericana de desarrollo rural territorial 2010-2030: ECADERT/ CAC, ECADERT, SICA [ECADERT], 2010) que constituyen la calidad de la vida dependen de la construcción de capacidades de participación ciudadana en las poblaciones de la región. Por lo tanto, adquieren especial importancia todas las manifestaciones y recursos al servicio de la educación, tanto formal como informal. Sin la construcción de las competencias necesarias es imposible la participación ciudadana exitosa.

\section{¿Procesos de cambio, procesos de aprendizaje?}

A partir de estas realidades surge la necesidad de construir cambios de valores que construyan una lectura crítica de la realidad para motivar la responsabilidad en la ciudadanía. 
URL: http://www.una.ac.cr/educare

Esta construcción debe desatar una cadena de transformaciones en actitudes y valores que se concreten en conductas comprometidas con la calidad de la vida humana y de los ecosistemas que la sostienen.

El proceso de construcción de aprendizajes debe ser encarado desde variadas dimensiones y recursos. Uno de los primeros pasos debe ser la sensibilización de la audiencia mediante una lectura crítica de la necesidad de compromiso ante la vulnerabilidad social y ambiental, producto de sus acciones irreflexivas.

El enfoque pedagógico que más se ajusta a este objetivo es el de la educación popular:

Asume una posición epistemológica de carácter dialéctico, rechazando por tanto el viejo, tradicional y todavía "consagrado" marco positivista. En consecuencia, desarrolla una propuesta metodológica, pedagógica y didáctica basada en la participación, en el diálogo, en la complementación de distintos saberes. Y todo ello desde y [sic] para una opción política que ve el mundo desde la óptica de los marginados y excluidos y que trabaja en función de su liberación. (Núñez, 2005, p. 4)

La construcción participativa de una visión social de la comunidad, del territorio, nación o región es un proceso que permite construir una lectura crítica del presente, con proyección al futuro. Esta lectura reconoce la necesidad de sociedades incluyentes y equitativas. Valorar las ventajas del diálogo intercultural permite recoger los beneficios de la diversidad cultural. Las relaciones sociales, culturales y productivas equitativas son una exigencia de la sostenibilidad del bienestar y de la calidad de la vida. La inequidad social, de acceso a derechos humanos como el trabajo digno, están asociadas a incrementos de la violencia (Ortiz y Cummins, 2011).

La elaboración de la visión del futuro en el que queremos vivir, en forma consensuada, a largo plazo, permite reconocer las diversas dimensiones del bienestar humano y sus interrelaciones. Compartir este sueño es un paso ineludible para poder alejarse del bienestar asociado al consumo, concepto promovido e imperante en nuestra cultura. Es una oportunidad para alimentar la esperanza de que es posible construir el "inédito viable" del cual habla Paulo Freire (1993, p. 25).

A través de estos ejercicios, se busca construir la conciencia crítica (Freire, 2002) para poder establecer las correlaciones causales y las circunstancias de acuerdo como se dan los hechos en la realidad, tal como se experimentan. Se pretende alimentar a un ser humano sujeto de sus propios procesos; que reconoce su responsabilidad individual, social y ambiental en la construcción de la calidad de la vida que permite su bienestar.

A la sensibilización debe seguir la construcción de propuestas de cómo actuar, la propuesta contextualizada de conductas animadas con los nuevos valores. No solo es necesario 
sensibilizar, sino crear las condiciones que posibiliten pasar a la acción comprometida. Conocer el cómo hacer, de forma accesible y contextualizada, es algo que muchas veces se deja de lado y favorece la retórica que desmotiva a las personas.

Un aspecto importante reside en la construcción de esperanza, en concretar alternativas viables de bienestar equitativo, con respeto de la diversidad social y cultural. La construcción de redes en las que se compartan aprendizajes desde sus contextos alimentará estas esperanzas, al alentar la acción innovadora. Informarse y sentir que otros y otras están comprometidos con las mismas metas, desde contextos similares, pero diferentes, fortalece el compromiso. Es valioso promover los procesos con un sentido de "(...) movimiento socio-histórico, formando ciudadanos capaces de escoger los indicadores de calidad de su futuro (...)" (Gadotti, 1998, p. 10), que aspiran a transformarse en sujetos de los cambios que contribuyen a un proceso con trascendencia universal. Las redes hacen posible compartir las construcciones relevantes, significativas localmente, para interactuar con otros "contextos" facilitando el enriquecimiento mutuo (De Souza, 2005).

\section{¿Cómo construir estos cambios?}

La dificultad aparente de construir estos nuevos aprendizajes que permitan la recuperación, promoción y sostenibilidad de la calidad de la vida se desvanece cuando permitimos que afloren la sensibilidad y la intuición. Es importante sentir que el ser humano no está sobre el planeta para dominar sus recursos, sino para vivir en armonía con ellos. Respetar el bienestar de los ecosistemas, es respetar el propio bienestar. Existen recursos que no se podrán comprar con ningún dinero. A través de la sensibilidad hacia la vida, característica esencialmente humana, podemos alimentar la lectura crítica acerca del entorno. Construir mediante preguntas el análisis del presente y la proyección hacia el futuro. Al establecer estas visiones con criticidad, abrimos el camino hacia el reconocimiento de la interdependencia existente entre la calidad de vida humana y la calidad de vida de los ecosistemas que la hacen posible. De la misma forma se determina la necesidad de relaciones humanas equitativas e incluyentes como una condición inherente a una vida en armonía y sin violencia.

Como todo proceso de aprendizaje, esta construcción requiere del reconocimiento de los saberes previos. Conocer las motivaciones y contextos para proponer experiencias relevantes y pertinentes posibilita la construcción de cambios duraderos en las estructuras cognitivas de las personas. Solo los aprendizajes que tengan verdadero sentido emocional y lógico en los contextos específicos serán los que generen un verdadero compromiso de cambio (Rodríguez, 2004).

Es así como los saberes técnicos especializados deben entrar en diálogo con las realidades comunitarias concretas, en un proceso que reconozca, en cada caso, las motivaciones alimentadas por las necesidades humanas que entran en relación con las oportunidades contextuales para manifestarse en determinadas conductas. 


\section{Propuesta para diseñar los procesos, materiales y recursos que permitan construir la participación}

Las estrategias y políticas regionales contemplan cambios en las comunidades rurales. Estos cambios se relacionan con la construcción de una nueva ciudadanía participante en la gestión de los recursos que afectan directamente su bienestar. El diálogo en doble sentido -vertical y horizontal- entre los niveles locales, territoriales, nacionales y regional es esencial para garantizar la credibilidad y el sostén de todos los esfuerzos de integración y planificación sostenible del desarrollo rural en Centro América.

En consecuencia, es necesario acompañar las políticas de procesos de difusión y construcción de aprendizajes para desarrollar los cambios de valores y actitudes que construyan relaciones sociales y económicas equitativas e incluyentes. Estas relaciones permitirán el bienestar humano en forma sostenible - de la única forma posible- desde una relación de armonía con la Naturaleza y el reconocimiento de la interdependencia entre los seres humanos, los ecosistemas y el Universo. Esta perspectiva fue aprendida de las cosmovisiones de nuestras poblaciones indígenas, las cuales tienen aún mucho más que aportarnos, con su concepto de:

Vivir bien, es la vida en plenitud. Saber vivir en armonía y equilibrio; en armonía con los ciclos de la Madre Tierra, del cosmos, de la vida y de la historia, y en equilibrio con toda forma de existencia en permanente respeto. (Huanacuni, 2010, p. 32)

Estos cambios no se alcanzarán con una intervención. Para construir y difundir esta cosmovisión serán necesarios constantes y persistentes esfuerzos de reflexión en los distintos niveles de decisión.

Es frecuente que desde los ámbitos donde se construyen los saberes expertos se tome en cuenta su difusión hacia las comunidades. En algunos casos, el afán de comunicación no abre el reconocimiento de los saberes locales que sustentan las prácticas vigentes productivas, sociales y culturales. Eludir este paso compromete el éxito del enriquecimiento mutuo mediante el intercambio entre saberes locales y expertos.

La comunicación de los saberes expertos debe plantearse desde el contexto local, preguntándose acerca de las prácticas vigentes y las motivaciones a que responden. Estas prácticas son un producto de las cosmovisiones, necesidades humanas (no solo de subsistencia) y diálogo con su contexto social, cultural y natural.

En la actualidad se encuentran numerosas iniciativas que, en algunos casos, contemplan estrategias más o menos complejas de construcción de aprendizajes y pueden combinar diversos recursos y experiencias. Las tecnologías de información y comunicación (TIC) abren posibilidades que deben ser consideradas, si las condiciones del contexto local lo permiten. 
Una de las iniciativas más frecuentes consiste en la difusión de contenidos impresos, estos materiales suelen diseñarse para uso mediado o independiente. En algunos casos son parte de estrategias de difusión integradas por diversos medios. Esta forma, como cualquier otro esfuerzo de difusión de saberes, debe delimitar el objetivo: ¿qué cambios se desea que construya el público meta de la experiencia? Luego, de forma explícita, se debe definir a quién se dirige, cuáles son las características, intereses, prácticas, lenguaje, significados, cosmovisiones. Cuanto más directo sea el conocimiento de la audiencia meta, mayores serán las posibilidades de construir una experiencia exitosa de comunicación y cambio.

Como todo proceso educativo, deben delimitarse los contenidos de distinto tipo que se desean mediar: conceptuales, actitudinales, procedimentales. Con base en estos elementos se diseña la experiencia de aprendizaje propuesta. Se debe insistir en que los cambios de actitudes y valores que conducen a transformar prácticas no se logran con una única mediación. Aun cuando se observa el cambio en forma relativamente pronta, su perduración en el tiempo no es una cuestión asegurada.

Es importante señalar que las cuestiones que se contemplan en las estrategias y políticas regionales son complejas. Como tales, requieren un enfoque interdisciplinario. Es conveniente que las iniciativas de difusión consideren los aportes de un equipo interdisciplinario más allá de los expertos en política y desarrollo.

El proceso de diseño de los materiales que apoyen la difusión y construcción de aprendizajes debe apoyarse en un proceso de revisión y ajuste llevado a cabo entre todos los miembros del equipo. También es necesaria una experiencia exploratoria con la audiencia esperada. Estas actividades permiten enriquecer los materiales con los aportes de quienes, en definitiva, serán los protagonistas de los procesos.

\section{Un recurso valioso}

Es común que, con el afán de informar y motivar a la acción, se diseñen materiales impresos en los que los expertos en el tema resumen los contenidos importantes. Para cumplir las metas de difusión y motivación, es necesario pensar en un recurso que facilite los procesos de reflexión-educación colectiva. Los grupos de personas deben asumir el compromiso de pensar la realidad de manera colaborativa, deben pensar en el texto paralelo que responda a estos objetivos.

Con esta finalidad, el texto paralelo debe estar en función de garantizar la comprensión que lleve a la acción comprometida y promover, así, un lector activo que se pregunte y relacione el contenido con su contexto e intereses. El texto paralelo favorece operaciones cognitivas en quien usa el recurso y facilita el ejercicio de una lectura activa, al transferir al contexto los contenidos presentados. 
URL: http://www.una.ac.cr/educare

Este tipo de texto propone espacios de reflexión intercalados con los de información (Céspedes, 2000). En la medida en que la reflexión, individual o en grupo, se presente de forma familiar y favorezca la construcción de aplicaciones útiles para quien lee, se garantizará una mayor apropiación del contenido.

\section{Conclusiones}

Las amenazas y oportunidades que se abren en Centro América han promovido iniciativas de integración y diseño de estrategias y políticas de desarrollo integral regionales.

Alcanzar el bienestar de las comunidades humanas requiere de un cambio de visión ante la Naturaleza. Las estrategias y políticas regionales contemplan una visión de armonía entre los seres humanos, la naturaleza y el universo.

El éxito de las estrategias y políticas de integración y desarrollo en Centro América demanda la construcción de una ciudadanía participativa informada.

La participación efectiva de la ciudadanía da contenido y credibilidad a los esfuerzos de integración de la región.

La participación efectiva se construye a partir de la experiencia participativa. Esta participación da credibilidad a las decisiones políticas e instituciones democráticas.

Es necesario informar a la sociedad civil y a los miembros de las representaciones públicas en todos los niveles espaciales, acerca de las iniciativas de integración y desarrollo, así como de su alcance.

La participación informada y reflexiva en los procesos de construcción del bienestar humano y de los ecosistemas que lo hacen posible exige la difusión de información y construcción de aprendizajes.

El diseño de las experiencias de construcción de estos aprendizajes demanda un abordaje interdisciplinario y un ciclo de diseño -validación- rediseño.

Las experiencias efectivas que construyan cambios en las poblaciones involucradas se alcanzan mediante un diálogo entre los saberes expertos y los locales.

Los cambios requeridos para la construcción de un desarrollo integral respetuoso de la calidad de la vida en la región centroamericana exigen experiencias persistentes y coherentes que promuevan la lectura crítica de los valores y acciones de las comunidades.

La educación, en todas sus formas, debe asumir nuevos retos y articular esfuerzos que superan la visión tradicional que la limita a las iniciativas formales. Toda experiencia cuyo objetivo es generar cambios en las estructuras cognitivas y en los valores que dan sentido y 
orientación a la vida de los protagonistas fundamentando así sus decisiones y acciones, es una experiencia de aprendizaje. Como tal, amerita la definición de un enfoque pedagógico y de una mediación didáctica pertinente

\section{Referencias}

Céspedes, É. (2000). Estudiar mejor en la modalidad presencial y a distancia 1. Guía didáctica. Heredia, Costa Rica: Universidad Nacional y Universidad Ultrech.

De Souza, J. (2005). El poder de las redes y las redes de poder. Paradigmas emergentes para transformar la morfología social de sociedades y organizaciones en el contexto del cambio de época. San José, Costa Rica. Recuperado de http://es.scribd.com/doc/57211488/El-poderde-las-redes-y-las-redes-del-poder-Jose-de-Souza-Silva

Estrategia centroamericana de desarrollo rural territorial 2010-2030: ECADERT/ CAC, ECADERT, SICA. (2010). San José, Costa Rica: IICA. Recuperado de http://www.magfor.gob.ni/ descargas/planes/ECADERT.pdf

Freire, P. (1993). Pedagogía de la esperanza. México: Siglo XXI.

Freire, P. (2002). La educación como práctica de libertad. México: Siglo XXI.

Gadotti, M. (noviembre-diciembre, 1998). Pedagogía de la tierra y cultura de la sustentabilidad. Conferencia Continental de las Américas. Cuiabá, Matto Grosso: Consejo de la Tierra.

Grain. (marzo, 2010). Land Grabbing in Latin American [Apropiación de Tierras en América Latina]. Recuperado de http://www.grain.org/article/entries/3995-land-grabbing-inlatin-america

Grain. (febrero, 2012). Grain releases data set with 4000 global land grabs [Grain publica un conjunto de datos sobre la apropiación global de 4000 terrenos]. Recuperado de http:// www.grain.org/article/entries/4479-grain-releases-data-set-with-over-400-global-landgrabs

Huanacuni, F. (2010). Buen vivir/vivir bien. Filosofía, políticas, estrategias y experiencias regionales andinas. Perú: Coordinadora Andina de Organizaciones Indígenas (CAOI).

Instituto Interamericano de Cooperación para la Agricultura (IICA) y Dirección de Desarrollo Rural Sostenible. (2000). Nueva ruralidad (Serie: Documentos Conceptuales). San José, Costa Rica: IICA.

Instituto Interamericano de Cooperación para la Agricultura (IICA). (s. f.). Preguntas frecuentes sobre seguridad alimentaria. Costa Rica: Autor. Recuperado de http://www.iica.int/Esp/ conocimiento/actualidad/Documentos\%20Seguridad\%20Alimentaria/Preguntas\%20 frecuentes\%20sobre\%20la\%20seguridad\%20alimentaria.pdf 
Kliksberg, B. (domingo 13 de noviembre de 2011). ¿Cómo enfrentar la pobreza y la desigualdad? (Economistas ortodoxos en aprietos. El capital social se puso en marcha). Biblioteca Bernardo Kliksberg. Página 12, Suplemento especial, pp. I-IV. Recuperado de http://www. pagina12.com.ar/especiales/archivo/bernardo kliksberg/007-KLIKSBERG.pdf

Núñez, C. (enero-abril, 2005). Educación popular: Una mirada de conjunto. Decisio Educación Popular, 10. http://tariacuri.crefal.edu.mx/decisio/d10/sab1-3.php\#inicio

Ortiz, I. y Cummins, M. (2011). Global Inequality: Beyond the Bottom Billion. A Rapid Review of Income Distribution in 141 Countries. New York: United Nations Children's Fund (UNICEF). Recuperado de http://papers.ssrn.com/sol3/papers.cfm?abstract id=1805046

Rodríguez, M. L. (2004). La teoría del aprendizaje significativo. En A. J. Cañas, J. D. Novak y F. M. González (Eds.), Concept Maps: Theory, Methodology, Technology (Proc. of the First Int. Conference on Concept Mapping, pp. 1-10). Pamplona.

Soberanía Alimentaria. (s. f.). ¿Qué es la soberanía alimentaria? Recuperado de http://www. soberaniaalimentaria.net/definicion.php

\section{Cómo citar este artículo, según APA:}

Bartol, P. M. (2012). Participación de la población rural en los procesos territoriales de desarrollo Revista Electrónica Educare, 16(3), 93-104. Consultado de http://www.revistas.una.ac.cr/index.php/EDUCARE/issue/current

Nota: Para citar este artículo en otros sistemas puede consultar el hipervínculo "Como citar el artículo" en la barra derecha de nuestro sitio web.

http://www.revistas.una.ac.cr/index.php/EDUCARE/index 